\title{
The intra-rater and inter-rater reliability of a method measuring the sagittal curvature of the spine using surface topography
}

Erin Hannink ${ }^{\mathrm{a}}$, Karen Barker ${ }^{\mathrm{b}}$, Helen Dawes ${ }^{\mathrm{a}}$

${ }^{a}$ Oxford Brookes University, Faculty of Health and Life Sciences, Headington, Oxford, United Kingdom

${ }^{b}$ University of Oxford, Department of Orthopaedics, Rheumatology and Musculoskeletal Sciences, Oxford, United Kingdom

$\underline{\text { Introduction }}$

Spinal curvature in the sagittal plane is associated with poor health related outcomes with age, such as decreased mobility, respiratory problems and increased mortality [1]. The gold standard for measuring sagittal spine curvature is the modified Cobb angle obtained from a lateral radiograph of the spine [2]; however, it is expensive and employed restrictively due to radiation exposure. Other widely accepted noninvasive methods, such as the flexicurve and Debrunner's kyphometer, have been shown to have high reliability and moderate validity in the thoracic kyphosis measurement [3], indicating space exists for a more robust, accurate non-invasive measure tool. New time-of-flight technology of the Microsoft Kinect Sensor V2 has the potential to provide more robust and accurate analysis of the curvature of the spine. This surface topography sensor can reconstruct a three-dimensional image of the back offering a relatively quick, inexpensive method to measure the spinal alignment. The reliability of this technology applied to spinal curvature in the sagittal plane has not been tested in an adult and older aged population.

\section{$\underline{\text { Research question }}$}

The aim of the study was to determine the intra-rater and inter-rater reliability of sagittal spine curvature measurement using the Microsoft Kinect Sensor V2.

\section{Methods}

Thirty-seven healthy individuals ( $57 \%$ female; age $=51.7 \pm 20.6(18-79) ; \mathrm{BMI}=24.9 \pm 3.3)$ participated in a cross-sectional study. Rater 1 captured three images of the spine and a second rater, blinded to the results of the first rater, captured three images of the same participant. Thoracic kyphosis was measured by calculating the maximum height and length of the region e.g. kyphosis index = (height) / (length from C7 to L1) (Fig. 1). 

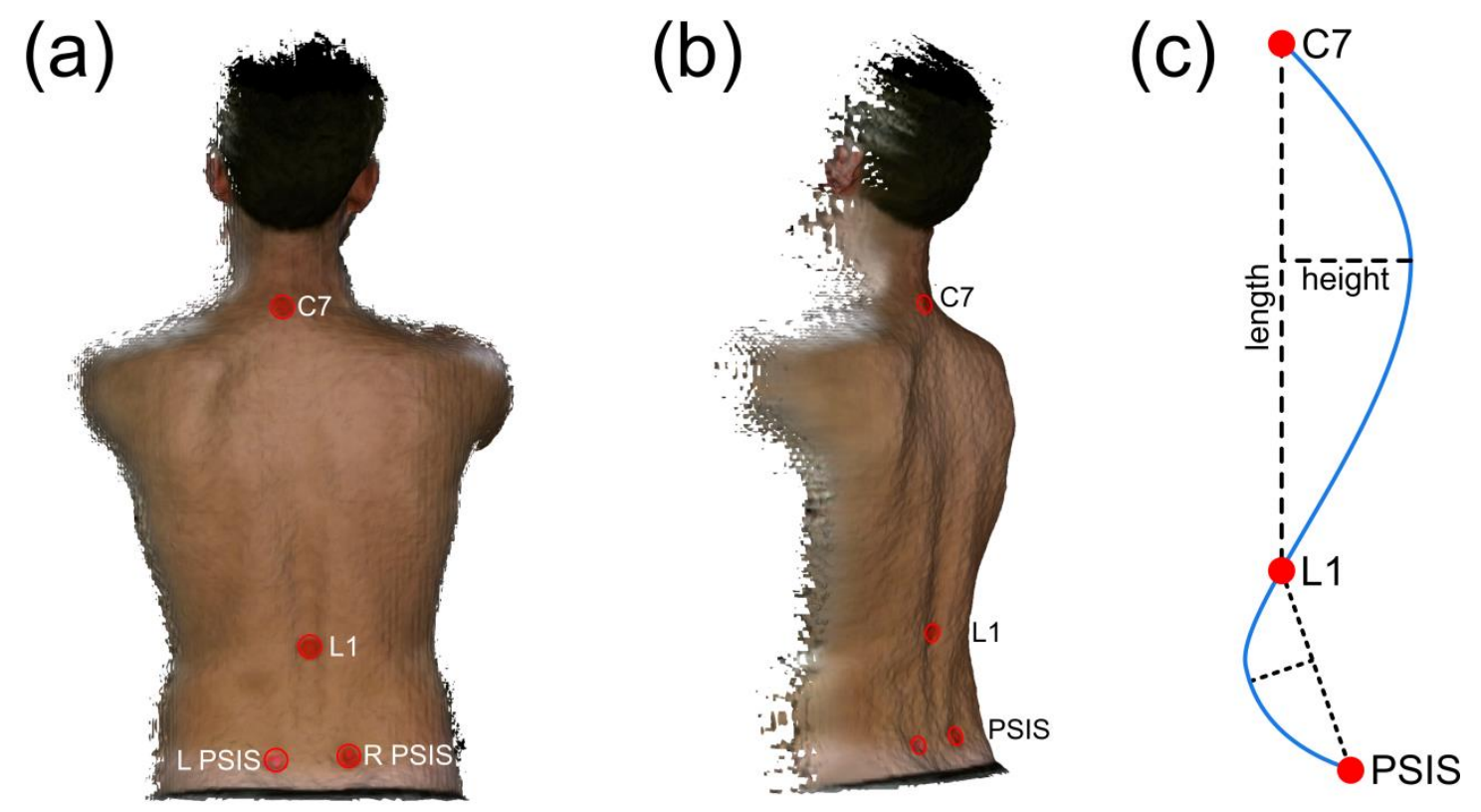

Fig. 1. (a) Reconstructed 3D image using Meshlab software from original view of kinect sensor which was placed behind the participant perpendicular to the coronal plane (b) Same image rotated to view the sagittal plane of the spine (c) Schematic of anatomical markers used to calculate kyphosis index (KI) of the spine: $\mathrm{KI}=$ height/length.

$\underline{\text { Results }}$

The Microsoft Kinect Sensor V2 showed excellent intra-rater reliability (ICC coefficient $(3,1)=0.960$, with $95 \% \mathrm{Cl}(0.926,0.979))$ and inter-rater reliability $(\operatorname{ICC}(2,1)=0.971$, with $95 \% \mathrm{Cl}(0.954,0.984))$ in measuring the kyphosis index of the thoracic spine.

\section{Discussion}

These results suggest that the Microsoft Kinect Sensor V2 is a reliable tool for measuring thoracic kyphosis with a repeatable method. While there is a need to validate this method against the gold standard, it has shown to be a versatile technology with the potential to provide a more in depth and robust measurement of spinal curvature in the sagittal plane for possible application in both research trials and clinical use.

Keywords: Sagittal spine, Kinect, Surface topography

\section{References}

[1] W.B. Katzman, L. Wanek, J.A. Shepherd, D.E. Sellmeyer, Age-Related Hyperkyphosis: Its Causes, Consequences, and Management, J. Orthop. Sport. Phys. Ther. 40 (2010) 352-360. doi:10.2519/jospt.2010.3099.Age-Related.

[2] T. Vrtovec, F. Pernuš, B. Likar, A review of methods for quantitative evaluation of spinal curvature, Eur. Spine J. 18 (2009) 593-607. doi:10.1007/s00586-009-0913-0.

[3] G.A. Greendale, N.S. Nili, M.H. Huang, L. Seeger, A.S. Karlamangla, The reliability and validity of three non-radiological measures of thoracic kyphosis and their relations to the standing radiological Cobb angle, in: Osteoporos. Int., 2011: pp. 1897-1905. doi:10.1007/s00198-010-1422-z. 